\title{
Cultivo de vieiras em Santa Catarina: tecnologias utilizadas e influência de fatores ambientais
}

\author{
Guilherme Sabino Rupp ${ }^{1}$
}

\begin{abstract}
Resumo - Neste trabalho é apresentada uma síntese das atividades de cultivo da vieira Nodipecten nodosus em Santa Catarina, das tecnologias utilizadas e dos resultados de estudos sobre a influência dos fatores ambientais nas vieiras cultivadas. O cultivo é realizado em lanternas japonesas suspensas em sistema tipo long-line de superfície, no qual as vieiras atingem o tamanho comercial de 7 a $8 \mathrm{~cm}$ em cerca de um ano, e são comercializadas ao preço de aproximadamente $\mathrm{R} \$ 50,00$ dúzia-1 ${ }^{-1}$. Com base em resultados de pesquisas, são discutidos os principais fatores limitantes para a ampliação da produção de vieiras no Estado, tais como pouca disponibilidade de áreas com condições ambientais adequadas ao cultivo e são sugeridas propostas para superá-los.
\end{abstract}

Termos para indexação: moluscos bivalves; Nodipecten nodosus; maricultura

\section{Scallop culture in Santa Catarina: technologies used and influence of environmental factors}

\begin{abstract}
This paper presents a summary of the aquaculture activities with the scallop Nodipecten nodosus in Santa Catarina, the technologies used, and results of studies on the influence of environmental factors on cultivated scallops. The cultivation is carried out in Japanese-type lantern-nets suspended in surface long-lines, in which the scallops reach the commercial size of 7 to $8 \mathrm{~cm}$ in about a year, and are sold at a price of approximately $\mathrm{R} \$ 50,00$ dozen $^{-1}$. Based on research results, the main constraints for expanding the production of scallops in the State, such as the availability of areas with adequate environmental conditions are discussed and suggestions are made to overcome them.
\end{abstract}

Index terms: bivalve molluscs; Nodipecten nodosus; mariculture

\section{Introdução}

As vieiras são moluscos bivalves da família Pectinidae com ocorrência em todos os mares e oceanos e que apresentam grande importância ecológica e econômica, sendo alvo de importantes atividades de pesca e aquicultura em muitos países. No Brasil, estão descritas 15 espécies nativas (RIOS, 2009), sendo a maioria de pequeno tamanho (menores que $7 \mathrm{~cm}$ ) à exceção de Nodipecten nodosus e Euvola (=Pecten) ziczac.

O cultivo de vieiras em escala comercial teve início no Japão na década de 1960 com a espécie Mizuhopecten (=Patinopecten) yessoensis em Mutsu Bay, Prefeitura de Aomori. Nas décadas de 1980 e 1990, as técnicas de cultivo desenvolvidas foram disseminadas para outros países (SHUMWAY \& PARSONS, 2006).

A produção mundial de vieiras por meio de aquicultura, em 2017, foi de 2.185.243 toneladas (FAO, 2019), sendo a China o maior produtor mundial, com 92,6\% dessa produção. Em seguida vem o Japão com 6,2\%, o Peru com 0,55\%, e demais países, tais como, Rússia, Chile, Coréia, Canadá, Noruega, Brasil, entre outros, que dividem o restante $0,65 \%$ da produção.

\section{O cultivo de vieiras em San- ta Catarina}

As pesquisas visando ao desenvolvimento do cultivo de vieiras no Brasil tiveram início em Florianópolis, nos primeiros anos da década de 90 (RUPP, 1992; 1994; MANZONI, 1994).

Motivados pelo entusiasmo gerado pelo então recente estabelecimento do cultivo comercial de ostras e mexilhões em Santa Catarina, bem como pelo sucesso do cultivo de vieiras em outros países, buscou-se identificar uma espécie nativa de vieira que apresentasse potencial para aquicultura. Poucas informações existiam sobre o tema, além de trabalhos de taxonomia e paleontologia (RIOS, 1985; SMITH, 1991). Identificouse Nodipecten nodosus como a maior vieira do litoral brasileiro (até $18 \mathrm{~cm}$ ), com ocorrência descrita para águas ra- sas do litoral de Santa Catarina, e optouse por iniciar estudos com esta espécie. Outra vieira de importância comercial descrita para Santa Catarina, porém de menor tamanho e ocorrência em maior profundidade, é Euvola (=Pecten) ziczac (RIOS, 2009). Nas décadas de 70 e 80, esta espécie foi alvo da indústria pesqueira ao largo do litoral de Santa Catarina, Paraná e São Paulo, porém, devido à excessiva explotação, seus estoques foram dizimados de maneira irrecuperável (PEZZUTO \& BORZONE, 2004).

A etapa fundamental para o estabelecimento de qualquer atividade de aquicultura é a obtenção de formas jovens (sementes) em quantidades suficientes e de qualidade adequada para suprir a demanda dos produtores. No caso do cultivo de moluscos, esta etapa pode ser realizada através da captação natural, com uso de coletores de sementes, que são colocados no mar na época de reprodução da espécie, ou por produção em condições de laboratório.

Iniciou-se, então, um estudo sobre o ciclo reprodutivo e a viabilidade de captação de sementes de $N$. nodosus no

Recebido em 30/10/2019. Aceito para publicação em 18/03/2020.

1 Biólogo, Ph.D., Epagri/Centro de Desenvolvimento em Aquicultura e Pesca, Rod. Admar Gonzaga 188, Florianópolis, SC, 88010-970, e-mail: rupp@epagri. sc.gov.br 
ambiente natural, cujos resultados não foram promissores para propósitos de aquicultura (MANZONI et al., 1996). A baixa captação de sementes, associada às caraterísticas reprodutivas e populacionais dessa espécie, indicaram que a captação natural não seria viável para abastecer os cultivos, concluindo-se que a produção em laboratório seria a única maneira de viabilizar o aproveitamento de $N$. nodosus para aquicultura.

Estudos foram realizados para 0 desenvolvimento de tecnologia de produção de sementes de $N$. nodosus no Laboratório de Cultivo de Moluscos (LMM/UFSC) (RUPP \& POLI, 1994; BEM et al., 2001; RUPP et al., 2004a; RUPP et al., 2005; ZANETTE, 2007). Assim que superados os principais entraves para a produção de sementes em quantidades suficientes para abastecer produtores, tomou-se a iniciativa, em 2006, de fomentar a atividade em escala piloto e comercial em Santa Catarina.

Através de iniciativa conjunta entre Epagri e UFSC, foram feitos seminários sobre cultivo de vieiras para extensionistas e produtores de ostras e mexiIhões, e iniciou-se o fornecimento de sementes de $N$. nodosus para cultivo. A partir de então teve início a produção comercial de vieiras em pequena escala no Estado, a qual vem se mantendo até os dias atuais, porém com pouca evolução nos volumes de produção, que tem variado entre 5 e 37 toneladas nos últimos anos (EPAGRI, 2018). Alguns dos produtores de ostras e/ou mexilhões cultivam vieiras como um produto alternativo e de maior valor agregado, não sendo conhecidos atualmente produtores exclusivos de vieiras. Enquanto o preço médio das ostras comercializadas pelos produtores em 2017 foi de aproximadamente $R \$ 7,70$ dúzia $^{-1}$, as vieiras atingiram o preço médio de $\mathrm{R} \$ 47,50$ dúzia-1 (EPAGRI, 2018).

Cultivos comerciais de vieiras vêm sendo, ou já foram realizados, nos municípios de Florianópolis, Palhoça, São José, Governador Celso Ramos, Bombinhas, Porto Belo, Balneário Camboriú e Penha. Houve casos de iniciativas de cultivos voltados exclusivamente à produção de vieiras no início desta década, os quais, infelizmente, tiveram curta existência. Os motivos de encerramento das atividades foram atribuídos, em alguns casos, a mortalidades causadas por episódios de baixa salinidade e, em outros, a questões administrativas e gerenciais, tais como falta de estrutura para comercialização.

\section{Tecnologia de cultivo}

A tecnologia de cultivo de $N$. nodosus em Santa Catarina envolve diversas etapas que incluem maturação de reprodutores, larvicultura, assentamento e berçário primário, realizadas em laboratório, seguidas das etapas de berçário secundário, cultivo intermediário e cultivo final ou engorda, realizadas no mar (RUPP \& PARSONS, 2016). Neste informativo técnico serão abordados alguns aspectos das etapas de cultivo berçário, intermediário e final, que são consideradas críticas para o sucesso da produção de vieiras.

\section{Cultivo Berçário}

Ao final da larvicultura, as larvas de $N$. nodosus são transferidas para o sistema de assentamento, ainda em laboratório, onde são colocadas em contato com coletores, constituídos por telas de monofilamento (Netlon ${ }^{\circ}$ ) para a fixação larval. Decorridas aproximadamente 2 a 3 semanas nesse sistema, quando atingem um tamanho médio de cerca de 0,5 - $1 \mathrm{~mm}$, os coletores podem ser transferidos para o mar, onde é realizada a segunda etapa do berçário (RUPP et al., 2005). Para o transporte, as bolsas coletoras são colocadas em sacos plásticos com água marinha filtrada, que por sua vez são acondicionados em caixas de isopor e levados ao local de cultivo. $O$ crescimento e a sobrevivência das sementes dependem das condições ambientais do local para onde são transportadas (IWERSEN et al., 2007). A taxa de crescimento das sementes em coletores colocados no mar da região de Porto Belo, por exemplo, varia entre $0,045 \mathrm{~mm} /$ dia no inverno, quando a temperatura é inferior a $20^{\circ} \mathrm{C}$, até $0,149 \mathrm{~mm} /$ dia no verão, quando a temperatura é superior a $25^{\circ} \mathrm{C}$. As taxas de crescimento podem ser descritas pela equação: $\mathrm{TCD}=65,69$ $+0,46 \mathrm{Te}-2,37 \mathrm{~S}-1,97 \mathrm{Tu}+0,88 \mathrm{TPM}$; ( $\mathrm{TCD}=$ taxa de crescimento diária, $\mathrm{Te}=$ temperatura, $\mathrm{S}=$ salinidade, $\mathrm{Tu}=$ turbidez e TPM= matéria particulada total) (RUPP et al., 2005). Este trabalho também demonstrou que, quando transferidas para o mar, as sementes podem crescer a uma velocidade cerca de 4 vezes maior do que aquelas mantidas em laboratório. A taxa de recuperação de sementes mantidas no mar por até 8 semanas situa-se entre 40 e $60 \%$. Nessa fase, a limpeza da malha externa dos coletores é muito importante, pois estas facilmente colmatam devido ao acúmulo de sedimentos, impedindo o fluxo de água e, consequentemente, de oxigênio e alimento para as sementes em seu interior. Deve-se também evitar excessiva agitação dos coletores para evitar o destacamento das sementes e consequente perda ou mortalidade.

Após um período de aproximadamente 6 a 8 semanas no mar, dependo das condições do local e da estação do ano, as sementes atingem um tamanho médio entre 5 e $15 \mathrm{~mm}$, quando podem ser destacadas dos coletores. As sementes devem, então, ser selecionadas por tamanho e as menores devem ser transferidas para lanternas berçário (malha de 2 a $4 \mathrm{~mm}$ ), ou caixas flutuantes (maIha de $2 \mathrm{~mm}$ ), onde apresentam maior crescimento (RUPP et al., 2009a). Já as sementes com cerca de 10 a $15 \mathrm{~mm}$ podem ser transferidas para lanternas de cultivo intermediário (malha de $6 \mathrm{~mm}$ ).

\section{Cultivo intermediário e engorda}

Em Santa Catarina as vieiras são cultivadas em sistema suspenso-flutuante, sendo utilizadas as mesmas estruturas onde são cultivadas ostras, ou seja, long-lines e lanternas japonesas.

Após a etapa de cultivo intermediário (cerca de 3 a 4 meses), quando atingem um tamanho médio aproximado de $30-35 \mathrm{~mm}$, as vieiras passam para a etapa de engorda ou cultivo final, quando podem ser usadas lanternas com abertura de malha de 15 , 20 e $25 \mathrm{~mm}$, sucessivamente, conforme avança o cultivo. A frequência de manejo, ou seja, a troca de lanternas e a redução de densidade, varia conforme o local, mas de maneira geral, deve ser realizada a cada 40 a 60 dias. As densidades devem ser ajustadas para que as vieiras ocupem cerca de $50 \%$ da área do piso da lanterna. Após cerca de 6 a 8 meses de cultivo final, as vieiras atingem tamanho médio que, dependendo do local, pode variar de 7 a $8 \mathrm{~cm}$ de altura de concha, e peso do músculo adutor de 8 a 12g.

O tamanho mínimo considerado. 
comercial para as vieiras situa-se entre 50-60mm, e peso de músculo adutor, seu principal atrativo gastronômico, superior a 6g. Entretanto, quanto maior o tamanho da vieira e o peso do músculo, maior é o seu valor comercial.

\section{Influências de fatores ambientais}

A maioria das áreas atualmente licenciadas para cultivo de moluscos, demarcadas pelos Planos Locais de Desenvolvimento da Maricultura de Santa Catarina - PLDM (SEAP/PR, 2008), localiza-se no interior, ou próximo às baías, muitas destas podendo ser influenciadas por águas estuarinas devido à proximidade de rios, e, em sua maioria, têm baixa profundidade. Estas áreas apresentam, também, alta concentração de material particulado inorgânico (MPI) em suspensão (FERREIRA et al., 2006), podendo ser afetadas por episódios de baixa salinidade (RUPP et al., 2009b). Em função da baixa profundidade, os long-lines utilizados ficam na superfície da água e, portanto, sujeitos à ação das ondas. Para cultivo de vieiras seria mais adequado o uso de long-lines cuja linha principal situa-se abaixo da superfície, minimizando a movimentação das lanternas causada pela ação das ondas. Estes, no entanto, só podem ser utilizados em áreas com maior profundidade.

As vieiras apresentam as brânquias (estruturas responsáveis pela respiração, retenção e seleção das partículas de alimento) com anatomia funcional significativamente diferente daquelas existentes em ostras e mexilhões (BENINGER et al., 1994). Nas vieiras estão ausentes os cílios látero-frontais e, com isso, estas têm menor eficiência na captura de alimento (fitoplâncton) em ambientes com alta concentração de MPI. Concentrações de MPI acima de $78 \%$ prejudicam o crescimento de vieiras (WALLACE \& REINSNES, 1985), incluindo N. nodosus (RUPP, 2007).

As taxas de crescimento e sobrevivência variam significativamente conforme o local e a profundidade de cultivo. Na Baía da Armação do Itapocoroy (município de Penha), após 10 meses de cultivo em um local com cerca de $12 \mathrm{~m}$ de profundidade, e utilizando-se long-line de subsuperfície, as vieiras atingiram aproximadamente $7 \mathrm{~cm}$ de altura mé- dia e cerca de $10 \mathrm{~g}$ de peso do músculo adutor, quando cultivadas na profundidade de $3 \mathrm{~m}$. Já as vieiras cultivadas a $10 \mathrm{~m}$ apresentaram, no mesmo período, crescimento significativamente menor, atingindo altura média de $6,5 \mathrm{~cm}$, com o peso de músculo não ultrapassando $6 \mathrm{~g}$ (RUPP, 2007). A principal diferença entre estas profundidades foi a MPI, a qual foi significativamente mais alta a $10 \mathrm{~m}$. Resultado similar foi encontrado por Rupp et al. (2004b) na região de Porto Belo, onde pré-sementes de $N$. nodosus apresentaram menor crescimento a $10 \mathrm{~m}$, em períodos quando se verificou maior concentração de MPI nessa profundidade. Em ambas as profundidades, a sobrevivência situou-se em torno de $80 \%$.

Nas baías Norte e Sul, na região de Florianópolis, por outro lado, as vieiras atingem o tamanho médio aproximado de $5,5 \mathrm{~cm}$ (altura da concha) e cerca de $5 \mathrm{~g}$ de peso do músculo adutor, em cerca de 10 meses de cultivo. O prosseguimento do cultivo por maior período não resulta em aumento do peso do músculo ou da concha, indicando que o estresse ecofisiológico nesse ambiente impede que as vieiras atinjam maior tamanho. Nessas áreas, as vieiras estão sujeitas à alta taxa de mortalidade devido a episódios de baixa salinidade e são alvo de intensa fixação de organismos epibiontes (biofouling) que podem causar danos às conchas e prejudicar o crescimento.

Rupp \& Parsons (2004) demonstraram que a salinidade letal média (LC 50 - 48h) para N. nodosus juvenis e adultos situa-se em torno de 23\%. Entretanto, em salinidades inferiores a $29 \%$ as vieiras já apresentam estresse fisiológico. Esse estudo também determinou que a temperatura letal média (LC 50 - 48h) situa-se em $29,8^{\circ} \mathrm{C}$ e as temperaturas ideais para cultivo de juvenis e adultos situa-se entre 23 e $27^{\circ} \mathrm{C}$.

\section{Considerações finais e recomendações}

Muitos estudos já foram realizados em Santa Catarina para subsidiar a produção de sementes de vieiras em laboratório e para definição de tecnologias para as distintas etapas de cultivo no mar, permitindo o estabelecimento dessa atividade em pequena escala co- mercial.

Atualmente, a disponibilidade de sementes não tem sido um fator limitante ao desenvolvimento da atividade. Porém, a ampliação da produção demandará a utilização de áreas de cultivo com condições ambientais mais adequadas aos requerimentos ecofisiológicos de $N$. nodosus. Em condições ambientais propícias, $N$. nodosus apresenta alta sobrevivência, podendo atingir tamanho comercial em cerca de um ano após a etapa de berçário, com altura de concha superior a $8 \mathrm{~cm}$ e peso do músculo superior a $10 \mathrm{~g}$. Entretanto, a maioria das áreas atualmente concedidas para cultivo de moluscos em Santa Catarina não permite aproveitar todo o potencial que as vieiras apresentam devido às características ambientais subótimas para essa espécie.

Dentre as áreas atualmente disponíveis para maricultura no Estado, devem ser utilizadas para vieiras apenas aquelas que apresentem características ambientais mais adequadas a seu cultivo, tais como: profundidade superior a $8 \mathrm{~m}$, percentagem de matéria inorgânica em suspensão inferior a $80 \%$, salinidade superior a $29 \%$ e temperaturas que não excedam $29^{\circ} \mathrm{C}$ por períodos prolongados. Caso novas áreas venham a ser licitadas para cultivo de moluscos em Santa Catarina, sugere-se que, além da qualidade microbiológica da água, esses parâmetros sejam considerados.

A identificação e a utilização de áreas com condições ambientais adequadas para cultivo de vieiras, bem como a demarcação de novas concessões marinhas, poderão permitir a ampliação do número de produtores de $N$. nodosus proporcionando o aumento da produção e um melhor aproveitamento do grande potencial que esta espécie apresenta para aquicultura. A existência de tecnologia de cultivo, o alto valor comercial, o cativante sabor e o mercado crescente, fazem com que o cultivo de vieras possa se tornar uma importante atividade, gerando relevantes benefícios econômicos e sociais no litoral catarinense.

\section{Referências}

BEM, M.M.; RUPP, G.S.; VANDENBERGHE, J.; SORGELOOS, P.; FERREIRA, J. Effects of marine bacteria on larval survival of the tropical scallop Nodipecten nodosus (Linnaeus, 
1758). In: INTERNATIONAL PECTINID WORKSHOP, 13., 2001, Coquimbo, Chile. Book of Abstracts [...] Coquimbo, Chile, 2001. p.111113.

BENINGER, P.G.; DWIONO, S.A.P.; LE PENNEC, M. Early development of the gill and implications for feeding in Pecten maximus (Bivalvia:Pectinidae). Mar. Biol., v.119, p.405-412. 1994.

EPAGRI. Síntese anual da agricultura de Santa Catarina 2017-2018. Florianópolis: Epagri/Cepa, 2018. p. 168-170.

FAO. Global Aquaculture Production 19502017. Fishery Statistical Collections, Fisheries and Aquaculture Department. Disponível em: <http://www.fao.org/fishery/ statistics/ global-aquaculture-production/es $>$. Acesso em: 9 abr. 2019.

FERREIRA, J.F.; BESSEN, K.; WORMSBECHER, A.G.; SANTOS R.F. Physical-chemical parameters of seawater mollusc culture sites in Santa Catarina - Brazil. J. Coast. Res., v.39, p.1122-1126, 2006.

IWERSEN, L.H.; RUPP, G.S.; MAES, G.; CANOZZI, M. B.; ZANETTE, G. B. Cultivo berçário de Nodipecten nodosus (Linnaeus, 1758) em dois locais do litoral de Santa Catarina. In: Congresso Latino-Americano de Ciências do Mar, 12., 2007, Florianópolis. Anais [...] Florianópolis, 2007. Disponível em: aoceano.org.br/downloads/colacmar2007 - artigo 4001870.

MANZONI, G.C. Aspectos da biologia de Nodipecten nodosus (Linnaeus, 1758) (Mollusca: Bivalvia), nos arredores da Ilha do Arvoredo (Santa Catarina - Brasil), com vista à utilização na aquicultura. 1994. 98f. Dissertação (Mestrado em Aquicultura) - Universidade Federal de Santa Catarina, Florianópolis, SC, 1994.

MANZONI, G.C.; POLI, C.R.; RUPP, G.S. Período reproductivo del pectinido Nodipecten nodosus (Mollusca Bivalvia) en los alrededores de la Isla Arvoredo (270 17'S - 48ㅇ 22'W) - Santa Catarina, Brasil. In: Silva, A.; Merino, G. (ED.). Acuicultura en Latinoamérica. Coquimbo, Chile: Asociación Latinoamericana de Acuicultura, 1996. p.197-201.

PEZZUTO, P.R.; BORZONE, C.A. The collapse of the scallop Euvola ziczac (Linnaeus, 1758) (Bivalvia: Pectinidae) fishery in Brazil: changes in distribution and relative abundance after 23 years of exploitation. Brazilian Jour- nal of Oceanography. v.52, n.3-4, p.225-236, 2004.

RIOS, E.C. Seashells of Brazil. Rio Grande: Fund. Cidade do Rio Grande, Brasil, 1985 $328 p$.

RIOS, E.C. Compendium of Brazilian Seashells. Evangraf, Rio Grande, Brasil, 2009. $688 p$.

RUPP, G.S. Pectinid culture is being investigated in Brazil. Out of the Shell -Mollusc Culture Network Newsletter, IDRC, Canada. v.2, n.3, p.6-8. 1992.

RUPP, G.S. Obtenção de reprodutores, indução à desova, e cultivo larval e pós-larval de Nodipecten nodosus (Linnaeus, 1758) (Bivalvia: Pectinidae). 1994. 125f. Dissertação (Mestrado em Aquicultura) - Universidade Federal de Santa Catarina, Florianópolis, SC, 1994.

RUPP, G.S. Cultivo da vieira Nodipecten nodosus em Santa Catarina: influência da profundidade, densidade e frequência de limpeza. Boletím Técnico n.135, Florianópolis: Epagri, 2007. 83p.

RUPP, G.S.; MAES, G.G.; FERREIRA, J.F. Floating trays: an unusual system for scallop nursery culture optimizes growth and survival of Nodipecten nodosus. In: INTERNATIONAL PECTINID WORKSHOP, 17., 2009, Book of Abstracts [...] Santiago de Compostela, 2009a. p.50.

RUPP, G.S.; PARSONS, G.J. Effects of salinity and temperature on the survival and byssal attachment of the lion's paw scallop Nodipecten nodosus at its southern distribution limit. J. Exp. Mar. Biol. Ecol., v.309, n.2, p.173-198, 2004.

RUPP, G.S.; PARSONS, G.J. Aquaculture of the scallop Nodipecten nodosus in Brazil. In: SHUMWAY, S.E.; PARSONS G.J. (Eds). Scallops: Biology, Ecology, Aquaculture and Fisheries. Amsterdam: Elsevier, 2016. p.9992017.

RUPP, G.S.; PARSONS, G.J.; THOMPSON, R.T.; DE BEM, M.M. Effect of depth and stocking density on growth and retrieval of the postlarval lion's paw scallop, Nodipecten nodosus. J. Shellfish Res., v.23, n.2, p.473-482, 2004b.

RUPP, G.S.; PARSONS, G.J.; THOMPSON,
R.J.; BEM, M.M. Influence of environmental factors, season and size at deployment on growth and retrieval of the postlarval Lion's Paw scallop, Nodipecten nodosus (Linnaeus, 1758) from a subtropical environment. Aquaculture, v.243, p.195-216, 2005.

RUPP, G.S.; POLI, C.R. Spat production of the sea scallop Nodipecten nodosus (Linnaeus, 1758), in the hatchery: initial studies in Brazil. Can. Tech. Rep. Fish. Aquat. Sci., n.1994, v.2, p.91-96, 1994.

RUPP, G.S.; THOMPSON, R.J.; PARSONS, G.J. Influence of food supply on post-metamorphic growth and survival of hatchery-produced lion's paw scallop Nodipecten nodosus (Linnaeus, 1758). J. Shellfish Res., v.23, n.1, p.5-13. 2004a.

RUPP, G.S.; ZANETTE, G.B.; BERCHT, M. Environmental influences on growth and survival of the scallop Nodipecten nodosus cultured at different sites in southern Brazil. In: INTERNATIONAL PECTINID WORKSHOP, 17., 2009, Book of Abstracts [...] Santiago de Compostela, 2009b. p.15-16.

SEAP/PR. SECRETARIA ESPECIAL DE AQÜICULTURA E PESCA DA PRESIDÊNCIA DA REPÚBLICA, Planos Locais de Desenvolvimento da Maricultura - PLDM's de Santa Catarina. Brasília, DF, 2008. 313p.

SHUMWAY, S.E.; PARSONS, G.J. (Eds.). Scallops: biology, ecology and aquaculture. Developments in Aquaculture and Fishery Science, v. 35, Amsterdam: Elsevier, 2006. 1460p.

SMITH, J.T. Cenozoic giant pectinids from California and the Tertiary Caribbean Province: Lyropecten, "Macrochlamis," Vertipecten, and Nodipecten species. U.S. Geological Survey Professional Paper, n.1391, 136p. 1991.

WALLACE, J.C.; REINSNES, T.G. The significance of various environmental parameters for growth of the iceland scallop Chlamys islandica (Pectinidae), in hanging culture. Aquaculture, v.44, p.229-242. 1985.

ZANETTE, G.S. Influência do tipo de coletor e do tempo de larvicultura na taxa de assentamento da vieira Nodipecten nodosus (L.) em laboratório. 2007, 33f. Dissertação (Mestrado em Aquicultura) - Universidade Federal de Santa Catarina, Florianópolis, SC. 2007. 